\title{
QUANTIZATION OF SYMPLECTIC ORBIFOLDS AND GROUP ACTIONS
}

\author{
ANA CANNAS DA SILVA AND VICTOR GUILLEMIN
}

\begin{abstract}
To a compact symplectic orbifold with a hamiltonian action of a group $G$ (and with other compatible data), we can associate a quantization space $V$ which is a representation of $G$. This paper reports on the decomposition of $V$ into irreducible representations of $G$ in terms of data at the fixed points, and on the semiclassical limit of this decomposition, for the case where $G$ is a compact connected semisimple Lie group.
\end{abstract}

\section{INTRODUCTION}

Let $(M, \omega)$ be a compact symplectic orbifold such that $[\omega]$ is the Chern class of a complex line bundle $L$ over $M$. Choose an almost complex structure, $J$, on $M$ compatible with $\omega$ (i.e. such that $(u, v) \mapsto$ $\omega(u, J v)$ is a positive metric), and choose a hermitian connection on $L$ with curvature given by $\omega$. To such data we can associate a first order elliptic differential operator, called the Dolbeault-Dirac operator $[8,6]$ :

$$
\not \partial: C^{\infty}\left(M ; \wedge^{0, \text { even }} T^{*} M \otimes L\right) \longrightarrow C^{\infty}\left(M ; \wedge^{0, \text { odd }} T^{*} M \otimes L\right) .
$$

Inspired by Bott [2], we define quantization of $(M, \omega)$, to be the (Ktheoretic) index of $\not$, i.e.

$$
Q(M, \omega):=\operatorname{kernel} \not \partial-\operatorname{cokernel} \not \partial \text {. }
$$

This index depends only on $(M, \omega)$ and not on the auxiliary data.

If a group $G$ acts on $M$, the action lifts to $L$ and preserves all data (in particular, the action must be hamiltonian [12]), then the quantization space becomes a linear representation of $G$. We will first address the following question: What is the decomposition of $Q(M, \omega)$ into irreducible representations of $G$ ?

As explained in this paper, not only does the answer depend exclusively on data at the fixed points, but also this answer can be given by a very explicit formula useful for computations. The prototype for

Date: November 1, 1998. 
this formula is a formula of Kostant [15] for multiplicities of group representations. The prototype for the proof is an observation of Cartier that we will now review.

From now on, let $G$ be a compact connected semisimple Lie group with a maximal torus $T$. Let $\rho: G \rightarrow \mathrm{GL}(V)$ be an irreducible representation with maximal weight $\lambda$. As a $T$-representation, $V$ breaks up into weight spaces

$$
V=\bigoplus_{\text {weights } \mu} V_{\mu}
$$

where $\operatorname{mult}(\mu):=\operatorname{dim} V_{\mu}$. Kostant [15] gave a formula for the multiplicity of a weight $\mu$ in $\rho$ :

$$
\operatorname{mult}(\mu)=\sum_{\omega \in W}(-1)^{\omega} \mathcal{P}(\omega(\delta+\lambda)-(\delta+\mu)),
$$

where $W$ is the Weyl group, $\delta=\frac{1}{2} \sum_{\alpha \in \Delta^{+}} \alpha, \Delta^{+}$is a chosen set of positive roots, and $\mathcal{P}(\nu)$ is the number of ways that $\nu$ may be partitioned into a sum of positive roots:

$$
\mathcal{P}(\nu):=\left\{\left(n_{\alpha} \in \mathbb{Z}_{0}^{+}\right) \mid \nu=\sum_{\alpha \in \Delta^{+}} n_{\alpha} \alpha\right\} .
$$

Cartier [7] showed that the Kostant formula is equivalent to the Weyl character formula, which states that

$$
\chi(\exp \xi)=\frac{\sum_{\omega \in W}(-1)^{\omega} e^{(\delta+\lambda)(\omega \xi)}}{e^{\delta(\xi)} \prod_{\alpha \in \Delta^{+}}\left(1-e^{-\alpha(\xi)}\right)},
$$

$\chi$ being the character of $\rho$ and $\xi \in \operatorname{Lie}(T)$. Cartier's simple argument starts by expanding the Weyl denominator in a power series, to obtain

$$
\frac{1}{\prod_{\alpha \in \Delta^{+}}\left(1-e^{-\alpha}\right)}=\sum_{\text {weights } \nu} \mathcal{P}(\nu) e^{-\nu},
$$

where $\mathcal{P}$ is the Kostant partition function. Now the Weyl formula can be written

$$
\sum_{\mu} \operatorname{mult}(\mu) e^{\mu(\xi)}=\sum_{\omega \in W} \sum_{\nu}(-1)^{\omega} \mathcal{P}(\nu) e^{(\delta+\lambda)(\omega \xi)} e^{-(\delta+\nu)(\xi)} .
$$

Formula (1.1) follows from equating the coefficients of $e^{\mu(\xi)}$.

The upshot of this argument is that formulas (1.1) and (1.2) are dual of each other by a Fourier expansion. This is less trivial than it sounds: There are several ways of expanding the Weyl denominator into a Fourier series, and for some of these expansions the coefficient of $e^{\nu}$ will be given by a divergent infinite sum. 
In $\S 4$ we obtain a formula (Theorem 1) resembling Kostant's for the multiplicity of a weight $\mu$ in $Q(M, \omega)$ for an action of a torus with the fixed points not necessarily isolated. We start from the equivariant index formula [1] modified for orbifolds [19, 8, 17], and apply a suitable Fourier expansion. Our proof uses the language of associated orbifolds explained in $\S$ A.3; Appendix A also reviews other relevant orbifold definitions. To ensure that each coefficient in the Fourier series is given by a convergent series, in $\S 3$ we define a polarization [10]. $\S 2$ describes how to handle an action of a compact connected semisimple Lie group by a combination of tricks.

In $\S 5$ we compute the semiclassical limit of the multiplicity formula (4.1): We replace the line bundle, $L$, by its $n$th tensor power, to get a $T$-invariant elliptic first order differential operator $\not^{(n)}$. Setting mult $^{(n)}(\mu)$ to be the multiplicity with which $\mu$ occurs as a weight of the representation of $T$ on

$$
\text { kernel } \not \partial^{(n)}-\operatorname{cokernel} \not \partial^{(n)},
$$

we show that, as the integer $n$ tends to infinity, the function of $\mu$ given by

$$
n^{-d} \operatorname{mult}^{(n)}(n \mu),
$$

where $d=\frac{1}{2} \operatorname{dim} M-\operatorname{dim} T$, tends to the Radon-Nikodym derivative for the Duistermaat-Heckman measure relative to the Lebesgue measure on the dual of the Lie algebra of $T$ : Theorem 2 of $\S 5$ and Theorem 3 of $\S 6$. The proof of Theorem 2 relies on complex analysis and on elementary results of invariant theory for finite groups; the proof of Theorem 3 relies on Fourier calculus and is analogous to the manifold proof in [6].

The case of torus actions on symplectic manifolds with only isolated fixed points was solved by the second author with Lerman and Sternberg [10]; their multiplicity formula was derived from the Atiyah-BottLefschetz formula by an argument along the lines of Cartier's. Later he and Prato [11] tackled the case of nonabelian actions (the argument in this paper is different from theirs and is an expansion of the remarks in [6]). The present paper is a continuation of [6] which contained the corresponding results for the case of manifolds. The extension to orbifolds is important since, not only are the generic reduced spaces of symplectic manifolds orbifolds, but also the generic reduced spaces of symplectic orbifolds are still orbifolds. Number-theoretic applications of multiplicity formulas for orbifolds can be found in [5]. 


\section{Quantization of Nonabelian Group Actions}

Let $\rho: G \rightarrow \mathrm{GL}(V)$ be a representation of a compact connected semisimple Lie group $G$ with a maximal torus $T$. The dimension of the space, $V^{G}$, of $G$-invariants is given by

$$
\begin{aligned}
\operatorname{dim} V^{G} & =\int_{G} \chi(g) d g \\
& =\frac{1}{|W|} \int_{T} \underbrace{\prod_{\operatorname{roots} \alpha}\left(1-e^{\alpha(\xi)}\right)}_{\sum_{\mu} c_{\mu} e^{\mu(\xi)}} \chi(t) d t \quad \text { where } \exp \xi=t \\
& =\frac{1}{|W|} \sum_{\mu} c_{\mu} \operatorname{dim} V_{\mu} .
\end{aligned}
$$

The middle equality follows from Weyl's integral formula (see, for instance, [3]), the others follow from orthogonality relations for characters, and the coefficients $c_{\mu}$ are constants. Hence, the dimension of the space of $G$-invariants is a linear combination of the multiplicities for the maximal torus with coefficients independent of the $G$-representation. ${ }^{1}$

Now let $V=Q(M, \omega)$ be the quantization space of a compact symplectic orbifold as described in $\S 1$, and let $\mathcal{O}$ be a coadjoint orbit of $G$ equipped with its canonical Lie-Poisson symplectic form, $\omega^{\mathcal{O}}$. The coadjoint action of $G$ is hamiltonian with moment map given by inclusion. By the Borel-Weil theorem, any irreducible complex $G$ representation, $\rho: G \rightarrow \mathrm{GL}\left(V^{\rho}\right)$ is the quantization space of a unique (integral) coadjoint orbit $\mathcal{O}$,

$$
Q\left(\mathcal{O}, \omega^{\mathcal{O}}\right) \simeq V^{\rho}
$$

The "shifting trick" [12] is the observation that

$$
\operatorname{dim}\left(Q\left(M \times \mathcal{O}, \omega-\omega^{\mathcal{O}}\right)\right)^{G}=\text { multiplicity of } \rho \text { in } Q(M, \omega) .
$$

We conclude that multiplicities for a nonabelian group action are linear combinations of multiplicities for the action of a maximal torus. From now on we restrict attention to actions of tori.

\footnotetext{
${ }^{1}$ We thank Michèle Vergne for showing us this trick.
} 


\section{Polarization}

Let $(M, \omega)$ be a compact symplectic orbifold such that $[\omega]$ is the Chern class of a complex line bundle $L$ over $M$. Suppose that a torus $T$ acts on $(M, \omega)$, and that this action is effective, lifts to $L$ and has a moment map $\Phi$. Choose an almost complex structure, $J$, on $M$ compatible with $\omega$ and choose a hermitian connection on $L$ with curvature given by $\omega$, both invariant by $T$.

The normal bundle, $N F$, to a connected component, $F$, of the fixed point set splits into a direct sum of vector subbundles

$$
E_{F, 1} \oplus \ldots \oplus E_{F, m}
$$

$m$ depending on $F$, such that the isotropy representation of $T$ on $E_{F, j}$ has a fixed rational weight, $\alpha_{F, j} \in \operatorname{Lie}(T)^{*}$, where $\alpha_{F, j} \neq \alpha_{F, k}$ for $j \neq k$. Since $T$ is compact, each $F$ is non-degenerate, in the sense that all $\alpha_{F, j} \neq 0$. Hence, we can polarize these weights as in [10] by choosing an element, $v$, of $\operatorname{Lie}(T)$ such that $\alpha_{F, j}(v) \neq 0$ for all $i, j$, and setting

$$
\alpha_{F, j}^{+}=\varepsilon_{F, j} \alpha_{F, j} \quad \text { where } \quad \varepsilon_{F, j}=\operatorname{sign} \alpha_{F, j}(v) .
$$

The polarized weights all lie in the half-space $\xi(v)>0$.

Let $n_{F, j}$ be half of the real rank of the vector bundle $E_{F, j}$, and let

$$
\alpha_{F}^{\#}=\sum_{\varepsilon_{F, j}=-1} n_{F, j} \alpha_{F, j} \quad \text { and } \quad n_{F}=\sum_{\varepsilon_{F, j}=-1} n_{F, j} .
$$

For every $m$-tuple of non-negative integers, $k=\left(k_{1}, \ldots, k_{m}\right)$, let $E_{F}(k)$ be the tensor product

$$
\left(\bigotimes_{j=1}^{m} \mathcal{S}^{k_{j}}\left(E_{F, j}^{+}\right)\right)^{*} \otimes\left(\bigotimes_{\varepsilon_{F, j}=-1} \wedge^{n_{F, j}}\left(E_{F, j}^{+}\right)\right)^{*}
$$

where $\mathcal{S}^{k_{j}}\left(E_{F, j}^{+}\right)$is the $k_{j}$-th symmetric power of $E_{F, j}^{+}$and

$$
E_{F, j}^{+}= \begin{cases}E_{F, j} & \text { if } \varepsilon_{F, j}=1 \\ E_{F, j}^{*} & \text { if } \varepsilon_{F, j}=-1\end{cases}
$$

\section{Multiplicity Formula for Orbifolds}

Given a weight $\mu$ of $T$, let $\Delta_{F}(\mu)$ be the convex polytope in $\mathbb{R}^{m}$ consisting of all $m$-tuples, $\left(s_{1}, \ldots, s_{m}\right), s_{i} \geq 0$, for which

$$
\Phi_{F}-\sum_{j} s_{j} \alpha_{F, j}^{+}=\mu
$$


where $\Phi_{F}$ is the value of the moment map $\Phi$ on the connected component $F$ of the fixed point set. In this expression $\alpha_{F, j}^{+}$and $\Phi_{F}$ are rational, whereas $\mu$ is integral. The fact that the $\alpha_{F, j}^{+}$'s are polarized (see $\S 4$ ) implies that $\Delta_{F}(\mu)$ is compact.

Theorem 1. The multiplicity with which $\mu$ occurs as a weight of the representation of $T$ on $Q(M, \omega)$ is equal to the sum

$$
\operatorname{mult}(\mu)=\sum_{F}(-1)^{n_{F}} \mathcal{N}_{F}(\mu)
$$

where

$$
\mathcal{N}_{F}(\mu)=\sum_{k \in \Delta_{F}\left(\mu-\alpha_{F}^{\#}\right)} \operatorname{KRR}\left(F, E_{F}(k) \otimes L_{F}\right)
$$

$\operatorname{KRR}\left(F, E_{F}(k) \otimes L\right)$ being the Kawasaki-Riemann-Roch number [14] of the orbifold vector bundle $E_{F}(k) \otimes L_{F}$ over $F$, with respect to the almost complex structure induced on $F$ by $J$.

Proof. The equivariant index theorem for orbifolds $[19,8]$ states that, for a generic $\xi$ in $\operatorname{Lie}(T)$ (generic in the sense that exp $\xi$ generates a dense subgroup of $T$ ), the trace of $\exp \xi$ on the (virtual) vector space $Q(M, \omega)$ is equal to the sum over the fixed point components, $F$, of "local traces", $\chi_{F}(\xi)$, whose definition we will now undertake.

Let $\widehat{F}$ be the orbifold associated to $F$, as described in $\S$ A.3; $\widehat{F}$ will in general have various connected components of possibly different dimensions, depending on the canonical stratification of $F$ (see $\S$ A.2). Let $L_{\widehat{F}}, E_{\widehat{F}, j}$ and $E_{\widehat{F}, j}^{+}$be the pull-backs of $\left.L\right|_{F}, E_{F, j}$ and $E_{F, j}^{+}$by the natural map $\mu: \widehat{F} \rightarrow F$; according to (3.1), the pull-back of the normal bundle $N F$ splits into $E_{\widehat{F}, 1} \oplus \ldots \oplus E_{\widehat{F}, m}$. These bundles over $\widehat{F}$ have canonical automorphisms as explained in $\S$ A.4, which we denote

$$
A_{F}:=A\left(L_{\widehat{F}}\right), \quad A_{F, j}:=A\left(E_{\widehat{F}, j}\right), \quad A_{F, j}^{+}:=A\left(E_{\widehat{F}, j}^{+}\right) .
$$

Finally, let $-2 \pi i \Omega_{F, j}$ be the curvature form associated with a hermitian connection on $E_{F, j}$, and we define $\Omega_{F, j}^{+}, \Omega_{\widehat{F}, j}$ and $\Omega_{\widehat{F}, j}^{+}$similarly for the bundles $E_{F, j}^{+}, E_{\widehat{F}, j}$ and $E_{\widehat{F}, j}^{+}$, respectively.

The local traces are then

$$
\begin{aligned}
& \chi_{F}(\exp \xi)= \\
& e^{\Phi_{F}(\xi)} \int_{\widehat{F}} \frac{1}{m_{\widehat{F}}} \cdot \frac{A_{F} \exp \left(\omega_{\widehat{F}}\right) \operatorname{Todd}(\widehat{F})}{D_{\widehat{F}} \prod_{j} \operatorname{det}\left(I-A_{F, j}^{-1} \exp \left(-\alpha_{F, j}(\xi) I-\Omega_{\widehat{F}, j}\right)\right)},
\end{aligned}
$$


where $\omega_{\widehat{F}}$ is the pull-back of the symplectic form $\omega$ to $\widehat{F}, m_{\widehat{F}}$ is the order function of $\widehat{F}$ (see $\S \mathrm{A} .2$ ) and $D_{\widehat{F}}$ is the associated characteristic form defined in $\S \mathrm{A} .4$ - the innocent looking $D_{\widehat{F}}$ is actually an elaborate characteristic form involving the normal bundles of the orbifold strata inside $F$.

If $\varepsilon_{F, j}=-1$, we rewrite the $j$ th term in the denominator in polarized form as

$$
\begin{aligned}
(-1)^{n_{F, j}} \operatorname{det} A_{F, j}^{-1} e^{-n_{F, j} \alpha_{F, j}(\xi)} & \operatorname{det} \exp \left(-\Omega_{\widehat{F}, j}\right) \cdot \\
& \operatorname{det}\left(I-A_{F, j} \exp \left(\alpha_{F, j}(\xi) I+\Omega_{\widehat{F}, j}\right)\right) .
\end{aligned}
$$

Let $E_{\widehat{F}}^{\#}$ be the line bundle

$$
\left(\bigotimes_{\varepsilon_{F, j}=-1} \wedge^{n_{F, j}}\left(E_{\widehat{F}, j}^{+}\right)\right)^{*}
$$

having curvature form $-2 \pi i \Omega_{\widehat{F}}^{\#}$ and canonical automorphism $A_{F}^{\#}=$ $\left(\prod_{\varepsilon_{F, j}=-1} \operatorname{det} A_{F, j}^{+}\right)^{-1}$. Since $\exp (\operatorname{trace} B)=\operatorname{det}(\exp B)$ for an endomorphism $B$, and the curvature form satisfies trace $\Omega(E)=\Omega\left(\wedge^{n} E\right)$, where $n$ is the rank of the vector bundle $E$, if we substitute (4.3) into (4.2), we can rewrite (4.2) in polarized form:

$$
\begin{aligned}
& \chi_{F}(\exp \xi)= \\
& (-1)^{n_{F}} e^{\left(\Phi_{F}-\alpha_{F}^{\#}\right)(\xi)} \int_{\widehat{F}} \frac{1}{m_{\widehat{F}}} \cdot \frac{A_{F} A_{F}^{\#} \exp \left(\omega_{\widehat{F}}+\Omega_{\widehat{F}}^{\#}\right) \operatorname{Todd}(\widehat{F})}{D_{\widehat{F}} \prod_{j} \operatorname{det}\left(I-\left(A_{F, j}^{+}\right)^{-1} \exp \left(-\alpha_{F, j}^{+}(\xi) I-\Omega_{\widehat{F}, j}^{+}\right)\right)} .
\end{aligned}
$$

By Lemma (B.1) of [6], we can expand (4.4) into an infinite trigonometric series

$$
\chi_{F}(\exp \xi)=(-1)^{n_{F}} \sum_{k} c_{k} e^{\left(\Phi_{F}+\alpha_{F}^{\#}-k_{1} \alpha_{F, 1}^{+}-\ldots-k_{m} \alpha_{F, m}^{+}\right)(\xi)}
$$

summed over all non-negative integer $m$-tuples, $k$, where $c_{k}$ equals

$$
\int_{\widehat{F}} \frac{1}{m_{\widehat{F}}} \cdot \frac{\left(\prod_{j} \operatorname{trace} \mathcal{S}^{k_{F, j}}\left(\left(A_{F, j}^{+}\right)^{-1} \exp \left(-\Omega_{\widehat{F}, j}^{+}\right)\right)\right) A_{F} A_{F}^{\#} \exp \left(\omega_{\widehat{F}}+\Omega_{\widehat{F}}^{\#}\right) \operatorname{Todd}(\widehat{F})}{D_{\widehat{F}}} .
$$


By definition of associated Chern character (see $\S$ A.4), formula (4.6) is simply

$$
\int_{\widehat{F}} \frac{1}{m_{\widehat{F}}} \cdot \frac{\widehat{\operatorname{Ch}}\left(E_{\widehat{F}}(k) \otimes L_{\widehat{F}}\right) \operatorname{Todd}(\widehat{F})}{D_{\widehat{F}}}
$$

which is the Riemann-Roch number of the orbifold line bundle $E_{F}(k) \otimes$ $L_{F}$ over $F$ as proved by Kawasaki [14].

On the other hand, for $\xi \in \operatorname{Lie}(T)$ the trace of $\exp \xi$ on the (virtual) vector space $Q(M, \omega)$ is equal to

$$
\sum_{\text {weights } \mu} \operatorname{mult}(\mu) e^{\mu(\xi)}
$$

and by comparing (4.5) with (4.7) one obtains the identity (4.1).

Remark. In the manifold case, we recover the result in [6] where the Kawasaki-Riemann-Roch number is the usual Riemann-Roch number:

$$
\mathrm{RR}\left(F, E_{F}(k) \otimes L_{F}\right)=\int_{F} \operatorname{Ch}\left(E_{F}(k) \otimes L_{F}\right) \operatorname{Todd}(F) .
$$

\section{Semiclassical Limit}

Let $n$ be a positive integer. Replacing the line bundle, $L$, by its $n$th tensor power, one gets, as in $\S 1$, a $T$-invariant elliptic first order differential operator $\not^{(n)}$. Denote by $\operatorname{mult}^{(n)}(\mu)$ the multiplicity with which $\mu$ occurs as a weight of the representation of $T$ on kernel $\not^{(n)}-$ cokernel $\not^{(n)}$, and let $d=\frac{1}{2} \operatorname{dim} M-\operatorname{dim} T$.

Theorem 2. As $n$ tends to infinity, the quantity $n^{-d}$ mult ${ }^{(n)}(n \mu)$ tends to

$$
\sum_{F \subseteq M^{T}}(-1)^{\sigma_{F}} \int_{\Delta_{F}(\mu)} \operatorname{Res}_{F}(s) d s,
$$

where $\operatorname{Res}_{F}(s)$ is the sum of residues of

$$
e^{\Sigma s_{j} z_{j}} \frac{1}{\left|\Gamma_{F}\right|} \int_{F} \frac{\exp \omega_{F}}{c_{F, 1}\left(z_{1}\right) \ldots c_{F, m}\left(z_{m}\right)},
$$

$\Gamma_{F}$ being the structure group of $F$ and $c_{F, j}(z)$ the Chern polynomial of $E_{F, j}^{+}$. 
Proof. By Theorem 1, mult ${ }^{(n)}(n \mu)$ is equal to the sum

$$
\sum_{F}(-1)^{n_{F}} \mathcal{N}_{F}^{(n)}(n \mu)
$$

where

$$
\mathcal{N}_{F}^{(n)}(n \mu)=\sum \int_{\widehat{F}} \frac{1}{m_{\widehat{F}}} \cdot \frac{\widehat{\operatorname{Ch}}\left(E_{\widehat{F}}(k) \otimes L_{\widehat{F}}^{n}\right) \operatorname{Todd}(\widehat{F})}{D_{\widehat{F}}}
$$

summed over all non-negative integral solutions, $k$, of the equation

$$
n \Phi_{F}-k_{1} \alpha_{F, 1}^{+}-\ldots-k_{m} \alpha_{F, m}^{+}+\alpha_{F}^{\#}=n \mu .
$$

(If we replace $L$ by $L^{n}$, we must replace $\omega$ by $n \omega, \Phi$ by $n \Phi$ and $A_{F}$ by $A_{F}^{n}$.) We set $\operatorname{dim} F=2 p$, and $\operatorname{dim} \Delta_{F}(\mu)=q$. By (5.3), we have

$$
n^{-d} \mathcal{N}^{(n)}(n \mu)=n^{-(d-p)} \sum_{k} \int_{\widehat{F}} n^{-p} \frac{1}{m_{\widehat{F}}} \cdot \frac{\widehat{\operatorname{Ch}}\left(E_{\widehat{F}}(k) \otimes L_{\widehat{F}}^{n}\right) \operatorname{Todd}(\widehat{F})}{D_{\widehat{F}}} .
$$

Lemma 5.5. Up to an error of order $O\left(\frac{1}{n}\right)$, the sum in (5.4) equals

$$
\sum_{k} \frac{1}{\left|\Gamma_{F}\right|} \sum_{g \in \Gamma_{F}} \rho_{F, 0}^{n}(g) \rho_{F}^{\#}(g) \int_{F} \exp \omega_{F} \prod_{j} \operatorname{trace} \mathcal{S}^{k_{j}}\left(\rho_{F, j}^{+}(g) \exp \left(-\frac{\Omega_{F, j}^{+}}{n}\right)\right),
$$

where $\rho_{F, 0}, \rho_{F}^{\#}, \rho_{F, j}^{+}$are the representations of the structure group $\Gamma_{F}$ of $F$ on the orbifold charts of $L, E^{\#}$ and $E_{j}^{+}$over $F$.

Proof. Let $\widehat{F}_{l}$ be a connected component of $\widehat{F}$ and let $2 p_{l}=\operatorname{dim} \widehat{F}_{l}$. With $\omega$ and $A_{F}$ replaced by $n \omega$ and $A_{F}^{n}$ in (4.6), the integrand in this expression can be expanded into a sum of terms of the form

$$
\pm \frac{1}{m_{\widehat{F}_{l}}} \cdot \frac{A_{F}^{n} A_{F}^{\#} \mathcal{A}\left(n \omega_{\widehat{F}}\right)^{r} \wedge \Omega_{i_{1}} \wedge \ldots \wedge \Omega_{i_{s}} \wedge\left(\Omega_{\widehat{F}}^{\#}\right)^{\nu} \wedge T^{\mu}}{D_{\widehat{F}_{l}}}
$$

where $\mathcal{A}$ is a factor involving the $A_{j}^{+}$'s, $\Omega_{i_{a}}$ is a coefficient of the curvature form $\Omega_{\widehat{F}, i_{a}}^{+}$, and $T^{\mu}$ is the component of degree $2 \mu$ of $\operatorname{Todd}\left(\widehat{F}_{l}\right)$. However, this term can only contribute to the integral if $r+s+\nu+\mu=$ $p_{l}$, in which case it can be rewritten as

$\pm \frac{1}{m_{\widehat{F}_{l}}} \cdot \frac{A_{F}^{n} A_{F}^{\#} \mathcal{A} n^{p_{l}} \omega_{\widehat{F}}^{r} \wedge\left(\Omega_{i_{1}} / n\right) \wedge \ldots \wedge\left(\Omega_{i_{s}} / n\right) \wedge\left(\Omega_{\widehat{F}}^{\#} / n\right)^{\nu} \wedge T^{\mu} / n^{\mu}}{D_{\widehat{F}_{l}}}$.

When multiplied by $n^{-p}$ (as in (5.4)), the terms in this sum for which $\nu$ or $\mu$ is positive or $p>p_{l}$ can be discarded since they contribute errors 
of order $O\left(\frac{1}{n}\right)$. We are left with the components of $\widehat{F}$ whose dimension is $2 p$. Such components are indexed by the conjugacy classes of $\Gamma_{F}$ (see $\S$ A.3). Hence the sum in (5.4) is equal to

$$
\sum_{k} \sum_{\gamma \in \operatorname{Conj}\left(\Gamma_{F}\right)} \frac{1}{m_{\widehat{F}_{\gamma}}} \int_{\widehat{F}_{\gamma}} \frac{A_{F}^{n} A_{F}^{\#} \exp \omega_{F_{\gamma}} \prod_{j} \operatorname{trace} \mathcal{S}^{k_{j}}\left(\left(A_{F, j}^{+}\right)^{-1} \exp \left(-\frac{\Omega_{\hat{F}, j}^{+}}{n}\right)\right)}{D_{\widehat{F}_{\gamma}}} .
$$

On the component $\widehat{F}_{\gamma}$, corresponding to $\gamma \in \operatorname{Conj}\left(\Gamma_{F}\right)$, we have

$$
A_{F}=\rho_{F, 0}(\gamma), \quad A^{\#}=\rho_{F}^{\#}(\gamma), \quad \text { and } \quad A_{j}^{+} \text {is conjugate to } \rho_{j}^{+}(\gamma)
$$

(we use the same symbol for a class function on a group and its induced function on conjugacy classes of that group). We also have $D_{\widehat{F}_{\gamma}}=1$ since $N_{\widehat{F}_{\gamma}}$ is trivial, $m_{\widehat{F}_{\gamma}}$ is the common order of the centralizers of elements in the conjugacy class $\gamma$, and any class function, $\chi$, satisfies

$$
\sum_{\gamma \in \operatorname{Conj}\left(\Gamma_{F}\right)} \frac{\chi(\gamma)}{m_{\widehat{F}_{\gamma}}}=\frac{1}{\left|\Gamma_{F}\right|} \sum_{g \in \Gamma_{F}} \chi(g) .
$$

Finally, as the natural immersions $\mu: \widehat{F}_{\gamma} \rightarrow F$ are bijective, the integral over $\widehat{F}_{\gamma}$ coincides with the integral over $F$.

Since the action of $T$ is effective, we have $q=m-\operatorname{dim} T$, and hence $d-p=q+\sum_{j=1}^{m}\left(n_{j}-1\right)$. By Lemma 5.5 and Lemma B.1, (5.4) is, up to an error of order $O\left(\frac{1}{n}\right)$, equal to

$$
n^{-q} \sum_{k} \mathcal{W}_{F, k} \operatorname{Res}\left[e^{\frac{k_{1}}{n} z_{1}+\ldots+\frac{k_{m}}{n} z_{m}} \int_{F} \frac{\exp \omega_{F}}{\prod_{j} \operatorname{det}\left(z_{j} I+\Omega_{F, j}^{+}\right)}\right]
$$

where the operator Res takes the sum of residues and the number $\mathcal{W}_{F, k}$ equals

$$
\frac{1}{\left|\Gamma_{F}\right|} \sum_{g \in \Sigma_{F}} \rho_{F, 0}^{n}(g) \rho_{F}^{\#}(g) \prod_{j}\left(\rho_{F, j}^{+}(g)\right)^{-k_{j}},
$$

$\Sigma_{F}$ being the abelian subgroup of $\Gamma_{F}$ consisting of the elements, $g$, for which each $\rho_{F, j}^{+}(g)$ is a scalar multiple of the identity. By the orthogonality relations for characters we know that

$$
\sum_{g \in \Sigma_{F}}\left(\rho_{F, 0}^{n} \rho_{F}^{\#} \prod_{j}\left(\rho_{F, j}^{+}\right)^{-k_{j}}\right)(g)=0
$$


unless $\left(\rho_{F, 0}^{n} \rho_{F}^{\#} \prod_{j}\left(\rho_{F, j}^{+}\right)^{-k_{j}}\right)$ is the trivial character of $\Sigma_{F}$, in which case this sum is $\left|\Sigma_{F}\right|$. Hence, (5.7) is equal to

$$
n^{-q} \sum_{k} \frac{\left|\Sigma_{F}\right|}{\left|\Gamma_{F}\right|} \operatorname{Res}\left[e^{\sum_{j} \frac{k_{j}}{n} z_{j}} \int_{F} \frac{\exp \omega_{F}}{\prod_{j} \operatorname{det}\left(z_{j} I+\Omega_{F, j}^{+}\right)}\right]
$$

summed over all $k$ satisfying both

$$
\Phi_{F}-\frac{k_{1}}{n} \alpha_{F, 1}^{+}-\ldots-\frac{k_{m}}{n} \alpha_{F, m}^{+}+\frac{\alpha_{F}^{\#}}{n}=\mu
$$

and

$$
\rho_{F, 0}^{n} \rho_{F}^{\#} \prod_{j}\left(\rho_{F, j}^{+}\right)^{-k_{j}}=\mathrm{id}
$$

in the character group of $\Sigma_{F}$. By representation theory for finite abelian groups, condition (5.9) is picking out a sublattice of order $\left|\Sigma_{F}\right|$ inside $\mathbb{Z}^{m}$. Therefore, as $n$ tends to infinity (5.8) tends to the integral

$$
\int_{\Delta_{F}(\mu)} \operatorname{Res}\left[e^{s z} \int_{F} \frac{1}{\left|\Gamma_{F}\right|} \cdot \frac{\exp \omega_{F}}{c_{1}\left(z_{1}\right) \ldots c_{m}\left(z_{m}\right)}\right] d s,
$$

where $c_{j}\left(z_{j}\right):=\operatorname{det}\left(z_{j} I+\Omega_{F, j}^{+}\right)$is the Chern polynomial of $E_{F, j}^{+}$.

Remark. When the dimension of $\Delta_{F}(\mu)$ is $q>0$ expression (5.8) converges pointwise to $(5.10)$ for all rational $\mu$. However, when $q=0$ it converges to (5.10) in a weak sense: for $\mu$ rational and any continuous test function $f$, the distribution (5.8) evaluated at $f$ tends to the evaluation of the distribution (5.10) at $f$, as $n$ tends to infinity.

\section{Duistermaat-Heckman Measure}

In [6] it was proved that, in the case of manifolds, the function of $\mu$ defined by (5.1) is the Radon-Nikodym derivative

$$
\frac{d \mu_{\mathrm{DH}}}{d \mu_{\text {Lebesgue }}}
$$

where $\mu_{\mathrm{DH}}$ is the Duistermaat-Heckman measure and $\mu_{\text {Lebesgue }}$ is the standard Lebesgue measure on $\operatorname{Lie}(T)^{*}$ (suitably normalized). The same is true for orbifolds:

Theorem 3. The piecewise polynomial function defined by (5.1) is the Radon-Nikodym derivative (6.1). 
The proof is analogous to that in [6]. Instead of the usual abelian localization formula, the orbifold version should be used [17], where the contributions of the fixed point components are affected by a factor equal to the inverse of the order of the orbifold structure group of that component [4].

\section{Appendix A. ORBifolds}

A.1. An $n$-dimensional orbifold, $M$, is a Hausdorff topological space, $|M|$, equipped with a complete atlas of orbifold charts, that is, a collection of triples $(\widetilde{\mathcal{U}}, \Gamma, \phi)$ where $\widetilde{\mathcal{U}}$ is a connected open subset of $\mathbb{R}^{n}, \Gamma$ is a finite group acting on $\widetilde{\mathcal{U}}$ by linear transformations, ${ }^{2}$ and $\phi: \widetilde{\mathcal{U}} \rightarrow|M|$ is a continuous $\Gamma$-invariant map inducing a homeomorphism from $\widetilde{\mathcal{U}} / \Gamma$ to $\mathcal{U}:=\phi(\widetilde{\mathcal{U}})$; satisfying appropriate compatibility conditions and such that the $\mathcal{U}$ 's cover $|M|$ and this collection of charts is maximal; more details and a review of orbifold differential geometry can be found in $[4,8,13,16,17,18]$. For instance, a smooth function on an orbifold is a collection of smooth invariant functions on each orbifold chart which agree on overlaps, and similarly for many other concepts.

A.2. Let $M$ and $N$ be two orbifolds with a continuous inclusion of the underlying topological spaces. Suppose that $M$ has an orbifold atlas where on each chart $(\widetilde{\mathcal{U}}, \Gamma, \phi)$ the pre-image of $N$, if not empty, is given by the intersection of $\widetilde{\mathcal{U}}$ with a linear subspace $V$ of $\mathbb{R}^{n}$. Let $\Gamma_{V}$ be the subgroup of those elements in $\Gamma$ whose action preserves $V$. Then $N$ is a suborbifold of $M$ if the collection $\left\{\left(\widetilde{\mathcal{U}} \cap V, \Gamma_{V},\left.\phi\right|_{\tilde{\mathcal{U}} \cap V}\right)\right\}$, together with the induced injections, forms an atlas of orbifold charts for $N$.

If $(\widetilde{\mathcal{U}}, \Gamma, \phi)$ is an orbifold chart for $M$, the structure group, $\Gamma_{p}$, of a point $p \in \mathcal{U}$ is the isotropy group of a pre-image of $p$ under $\phi ; \Gamma_{p}$ is defined up to isomorphism. The type of the structure group induces a canonical stratification of $M$ into suborbifolds. On each connected component of $M$, there is an open dense set of points for which the order of the structure group is minimal, called the principal stratum of $M$. When $M$ is connected, the structure group, $\Gamma_{M}$, of $M$ is the (abstract) isotropy group of its principal stratum; the order of $M$ is the order of $\Gamma_{M}$. When $M$ is not connected the orders of its connected components define the locally constant order function $m_{M}: M \rightarrow \mathbb{N}$.

\footnotetext{
${ }^{2}$ We assume that the set of all fixed points of $\Gamma$ has codimension at least two. We do not assume the action of $\Gamma$ to be effective. The result of the action of $g \in \Gamma$ on $u \in \mathcal{U}$ is denoted by $g \cdot u$.
} 
A.3. Given an orbifold $M$, the associated orbifold $[14,9] \widehat{M}$ has charts $(\widetilde{\mathcal{V}}, \Gamma, \psi)$ built as follows: For each chart, $(\widetilde{\mathcal{U}}, \Gamma, \phi)$, of $M$, let

$$
\widetilde{\mathcal{V}}=\{(u, g) \in \widetilde{\mathcal{U}} \times \Gamma \mid g \cdot u=u\} .
$$

$\Gamma$ acts on $\widetilde{\mathcal{V}}$ via $h \cdot(u, g)=\left(h \cdot u, h g h^{-1}\right)$. Let $\mathcal{V}=\widetilde{\mathcal{V}} / \Gamma$ be the space of orbits with projection $\psi: \widetilde{\mathcal{V}} \rightarrow \mathcal{V}$. The orbifold charts $(\widetilde{\mathcal{V}}, \Gamma, \psi)$ inherit the compatibility conditions from the $(\widetilde{\mathcal{U}}, \Gamma, \phi)$. As a set,

$$
\widehat{M}=\bigsqcup_{p \in M} \operatorname{Conj}\left(\Gamma_{p}\right)
$$

where $\operatorname{Conj}\left(\Gamma_{p}\right)$ is the set of conjugacy classes in $\Gamma_{p}$. For example, when $M$ is a teardrop orbifold (i.e. $M$ is homeomorphic to a sphere and has just one singular point, $p$, with structure group $\mathbb{Z} / n$ for some $n$ ), then $\widehat{M}$ has $n$ components: one component diffeomorphic to $M$ plus $n-1$ points each with structure group $\mathbb{Z} / n$, corresponding to $p$ paired with the nonzero elements of $\mathbb{Z} / n$. Associated orbifolds may be viewed as a bookkeeping device which allows for concise orbifold index formulas.

A.4. A rank $k$ orbifold vector bundle, $\pi: E \rightarrow M$, over an orbifold $M$ is a collection of $\Gamma$-equivariant rank $k$ vector bundles $\pi_{\tilde{\mathcal{U}}}: \widetilde{E}_{\mathcal{U}} \rightarrow \widetilde{\mathcal{U}}$ on each chart $(\widetilde{\mathcal{U}}, \Gamma, \phi)$, together with suitable compatibility conditions. The fibers $\pi^{-1}(p)$ are in general diffeomorphic to some quotient of a $k$-dimensional vector space by an the action of the structure group $\Gamma_{p}$. Additional structures on an orbifold bundle are defined as collections of $\Gamma$-invariant objects on each $\pi_{\widetilde{\mathcal{U}}}: \widetilde{E}_{\mathcal{U}} \rightarrow \widetilde{\mathcal{U}}$ agreeing on overlaps.

An orbifold vector bundle, $E \rightarrow \widehat{M}$, over an associated orbifold has a canonical automorphism, $A(E)$, given on each chart $(\widetilde{\mathcal{V}}, \Gamma, \psi)$ by the natural action of $g \in \Gamma$ on the fiber of $\widetilde{E}_{\mathcal{V}}$ above $(u, g) \in \widetilde{\mathcal{V}}[17]$.

Suppose that $M$ has an almost complex structure. Then the normal bundle, $N_{\widehat{M}}$, of the natural immersion $\mu: \widehat{M} \rightarrow M$ can be endowed with a hermitian connection; let $-2 \pi i \Omega\left(N_{\widehat{M}}\right)$ be its curvature. The associated characteristic form (appearing in the index formulas) is

$$
D_{\widehat{M}}=\operatorname{det}\left(I-A^{-1}\left(N_{\widehat{M}}\right) \exp \left(-\Omega\left(N_{\widehat{M}}\right)\right)\right) \text {. }
$$

The associated Chern character of a complex vector bundle $E \rightarrow \widehat{M}$ with curvature $-2 \pi i \Omega$ with respect to a hermitian connection is

$$
\widehat{\mathrm{Ch}}(E)=\operatorname{trace}(A(E) \exp \Omega) \text {. }
$$




\section{Appendix B. Integral Estimate}

Lemma B.1. Let $V$ be a d-dimensional complex vector space, and let $A, B$ be commuting elements of $\mathrm{GL}(V)$, with $A$ diagonalizable. Then

$$
n^{-(d-1)} \operatorname{trace} \mathcal{S}^{k}\left(A \exp \frac{B}{n}\right)= \begin{cases}\lambda^{k} \operatorname{Res}\left[\frac{e^{\frac{d+k-1}{n} z}}{\operatorname{det}(z-B)}\right]\left(1+O\left(\frac{1}{n}\right)\right) & \text { if } A=\lambda I \\ O\left(\frac{1}{n}\right) & \text { otherwise }\end{cases}
$$

where the $O\left(\frac{1}{n}\right)$ 's are uniform in $k$, and the operator Res takes the integral along a contour about the origin containing the zeroes of $\operatorname{det}(z-$ $B)$ and divides it by $2 \pi i$.

Proof. Without loss of generality, we can assume that $A$ and $B$ are simultaneously diagonalizable with eigenvalues $\lambda_{1}, \ldots, \lambda_{d}$ (of $A$ ) and $\mu_{1}, \ldots, \mu_{d}$ (of $B$ ), and that $e^{\mu_{1}}, \ldots, e^{\mu_{d}}$ are distinct. Let $C$ be a contour about the origin containing $\lambda_{1}, \ldots, \lambda_{d}$. For $n$ large enough, the left hand side of (B.2) is equal to the contour integral

$$
n^{-(d-1)} \frac{1}{2 \pi i} \int_{C} \frac{z^{d+k-1}}{\prod_{j}\left(z-\lambda_{j} e^{\mu_{j} / n}\right)} d z .
$$

The pole at $\lambda_{j} e^{\mu_{j} / n}$ has residue

$$
n^{-(d-1)} \frac{\left(\lambda_{j} e^{\mu_{j} / n}\right)^{d+k-1}}{\prod_{i \neq j}\left(\lambda_{j} e^{\mu_{j} / n}-\lambda_{i} e^{\mu_{i} / n}\right)}
$$

where

$$
\lambda_{j} e^{\mu_{j} / n}-\lambda_{i} e^{\mu_{i} / n}= \begin{cases}\lambda_{j} \frac{\mu_{j}-\mu_{i}}{n}\left(1+O\left(\frac{1}{n}\right)\right) & \text { if } \lambda_{j}=\lambda_{i} \\ \left(\lambda_{j}-\lambda_{i}\right)\left(1+O\left(\frac{1}{n}\right)\right) & \text { if } \lambda_{j} \neq \lambda_{i}\end{cases}
$$

There are $(d-1)$ factors in the denominator of (B.3). Therefore, if $A$ has at least two different eigenvalues, all residues (B.3) are of order $O\left(\frac{1}{n}\right)$. If $A=\lambda I$, then we express $n^{-(d-1)} \operatorname{trace} \mathcal{S}^{k}\left(\exp \frac{B}{n}\right)$ as in Theorem (B2) of [6].

By analyticity, (B.2) is valid for any endomorphism $B: V \rightarrow V$, not necessarily in $\mathrm{GL}(V)$. When $k$ is of order $O(n)$ (i.e. $k \rightarrow \infty$ as $n \rightarrow \infty$, but $\frac{k}{n}=O(1)$ ), (B.2) remains true if all eigenvalues of $A$ have absolute value at most 1 . 


\section{REFERENCES}

[1] Atiyah, M., Segal, G., The index of elliptic operators, II, Ann. of Math. 87 (1968) 531-545

[2] Bott, R., On induced representations, the mathematical heritage of Hermann Weyl (Durham, NC, 1987), 1-13, Proc. Sympos. Pure Math. 48, A.M.S., Providence, 1988.

[3] Bröcker, T., tom Dieck, T., Representations of Compact Lie Groups, Graduate Texts in Mathematics 98, Springer-Verlag, New York, 1995.

[4] Canas da Silva, A., Multiplicity Formulas for Orbifolds, Ph.D. thesis, M.I.T., 1996.

[5] Canas da Silva, A., Atiyah-Bott-Lefschetz for orbifolds and Dedekind sums, preprint, 1998.

[6] Canas da Silva, A., Guillemin, V., On the Kostant multiplicity formula for group actions with non-isolated fixed points, Adv. in Math. 123 (1996) $1-15$.

[7] Cartier, J., On H. Weyl's character formula, Bull. Amer. Math. Soc. 67 (1961) 228-230.

[8] Duistermaat, J. J., The Heat Kernel Lefschetz Fixed Point Formula for the Spin-c Dirac Operator, Birkhäuser, Boston, 1996.

[9] Farsi, C., K-theoretical index theorems for orbifolds, Quart. J. Math. Oxford 43 (1992) 183-200.

[10] Guillemin, V., Lerman, E., Sternberg, S., On the Kostant multiplicity formula, J. Geom. Phys. 5 (1988) 721-750.

[11] Guillemin, V., Prato, E., Heckman, Kostant, and Steinberg formulas for symplectic manifolds, Adv. Math. 82 (1990) 160-179.

[12] Guillemin, V., Sternberg, S., Geometric quantization and multiplicities of group representations, Invent. Math. 67 (1982) 515-538.

[13] Haefliger, A., Salem, É. Actions of tori on orbifolds, Ann. Global Anal. Geom. 9 (1991) 37-59.

[14] Kawasaki, T., The Riemann-Roch theorem for complex $V$-manifolds, Osaka J. of Math. 16 (1979) 151-159.

[15] Kostant, B., A formula for the multiplicity of a weight, Trans. Amer. Math. Soc. 93 (1959) 53-73.

[16] Lerman, E., Tolman, S., Hamiltonian torus actions on symplectic orbifolds and toric varieties, Trans. Amer. Math. Soc. 349 (1997) 4201-4230.

[17] Meinrenken, E., Symplectic surgery and the Spin ${ }^{c}$-Dirac operator, Adv. Math. 134 (1998) 240-277.

[18] Satake, I., On a generalization of the notion of manifold, Proc. N.A.S. 42 (1956) 359-363.

[19] Vergne, M., The equivariant index formula on orbifolds, Duke Math. J. 82 (1996) 637-652.

Department of Mathematics, University of California, Berkeley, CA 94720. E-mail address: acannas@math.berkeley.edu

Department of Mathematics, Massachusetts institute of TechnolOGY, CAMBridge, MA 02139. E-mail address: vwg@math.mit.edu 